\title{
Color Recognition System using Reconfigurable Architectures
}

\author{
G.Rakesh Chowdary ${ }^{1}$, B.Murali Krishna ${ }^{2}$, P.Srikanth $\operatorname{Reddy}^{1}$ Surendra Naveen Kumar, \\ Ravindra Somesh Kumar ${ }^{2}$ \\ ${ }^{1}$ Asst Professor, ${ }^{2}$ Assoc Professor Dept of ECE, KLEF, India rakeshchowdaryg @ kluniversity.in \\ ${ }^{2}$ U.G.Scoloar, Dept of ECE, KLEF, India someshkumarkondugari027@gmail.com
}

\begin{abstract}
Color recognition plays an important role in our daily life for human beings as well machines to detect Color applications like product manufacturing, packaging units, Skin Color recognition and Medical applications etc. Millions of Color combinations produced by basic Color s like Red, Green, and Blue. They are processed through camera and detected by system through different Color processing and sorting algorithms. In this paper an OV7670 Camera is used for Color recognition system (CRS) which processes the real image. CRS algorithm is designed with HDL and implemented on FPGA. In this system, the camera captures the real input values of the acquired Color and processes to Field Programmable Gate Array (FPGA). FPGA based CRS detects and then display the detected Color in Video Graphics Array (VGA) monitor. As FPGA provides high processing performance, more flexibility with low development cost and easily runtime reconfigurable.
\end{abstract}

Key words: Color Recognition system, OV7670, FPGA, VGA

\section{INTRODUCTION}

Color recognition technology takes gains more significance in artificial intelligence and machine learning applications for detection of an object Color and dimensions. The Purpose for the color Recognition is to separate the items relying upon their hues in an image. This discovery offers you the possibility of doing any sort of strategy regarding that object. For example, following marking in industry, skin color location as a pre-procedure of face discovery and picture examination in RGB space, a genuine color is divided to its fundamental color rates. In this color zone, Red, green, and blue hues are basics and each different hue can be gotten from their blends. In color screens for TV and PCs, RGB color space is utilized as a standard. For our framework, one among the advantages with respect to RGB region is, it is helpful for the preparation a segment of N.N. This preferred standpoint contributes framework at choosing level. Color recognition is another discovery innovation has played a critical job in Machine Vision and Artificial Intelligence. The color recognition of an item, the common procedure is to utilize a color sensor to gauge the Color components of the object in the RGB Color space. FPGA a reconfigurable hardware is used for algorithm processing. Since test outcomes are defense less to natural effects, this detection method ordinarily used in close range and speed less demanding system. Presently depending on software methods to accomplish Color recognition. The improvement of semiconductor innovation and rapid development of Digital signal processing architecture suitable for different processing applications.

\section{LITERATURE SURVEY}

Self-Adaptive Skin Segmentation in Color Images by Michal Kawulok, Jolanta Kawulok, Jakub Nalepa and Bogdan Smolka [1] in 2014 to describe introducing self-adaptive seeds, from which the pores and skin probability is propagated using the distance transform. Fast propagation-based skin regions segmentation in color images by Michal Kawulok [2] at 2013 to introduce a new technique for pores and skin regions segmentation which consists in spatial evaluation of pores and skin probability maps acquired using pixel-wise detectors. A new dynamic cellular learning automata-based skin detector by Ahmad Ali Abin, Mehran Fotouhi and Shohreh Kasaei [3] at 2009 Proposed a novel pores and skin detection algorithm that combines color and texture statistics of pores and skin with cellular studying automata to discover skin-like areas in shade images. Dynamic Skin Detection in Color Images for Sign Language Recognition by Michal Kawulok [4] at 2008 Geared toward detecting pores and skin in digital pics for Polish Sign Language reputation. A skin tone detection algorithm for an adaptive approach to steganography by Abbas Cheddad, Joan Condell, Kevin Curran, Paul Mc Kevitt [5] at 2009 Detecting human skin tone is of utmost significance in severa applications inclusive of, video surveillance, face and gesture recognition, human computer interplay, human pose modelling, image and video indexing and retrieval, image enhancing, automobile drivers' drowsiness detection, controlling users' surfing behaviour (e.G., browsing indecent 
sites) and steganography. Skin Segmentation Using Color Distance Map and Water-flow Property by $\mathrm{M}$. Abdullah-Al-Wadud, Oksam Chae [6] at 2008. A new technique for pores and skin place segmentation is proposed.

It makes use of shade distance map (CDM) and a set of rules primarily based at the assets of glide of water. Automatic Face Detection Based on Chrominance Components Analysis by M. Beniak, J. Pavlovicova and M. Oravec [7] in 2008. This paper describes the method to face localization task. A Novel Skin Tone Detection Algorithm for Contraband Image Analysis by Abhishek Choudhury, Marcus Rogers, Blair Gillam Purdue Cyber Forensics Lab Keith Watson [8] in 2008. This paper examines skin tone detection algorithms utilized by first responder forensic equipment including File Hound. File Hound is a "subject analysis" software utility this is presently being used by over 100 law enforcement groups, both internationally and domestically. Image based Face Recognition Issues and Method by Wen Yi Zhao and Rama Chellappa [9] in 2008. As one of the maximum a success applications of photograph analysis and understanding face popularity has recently received significant attention especially in the course of the beyond several years. Spatial-based skin detection using discriminative skin-presence features by Michal Kawulok, Michal Kawulok and Jakub Nalepa [10] in 2014. Proposed a new technique for skin detection in color pictures which is composed in spatial evaluation the use of the introduced texture-based totally discriminative pores and skin-presence features. Dynamic approach for real-time skin detection by Sara Bilal, Rina Akmeliawati, Memo Jimi E. Salami and Amir A. Shafie [11] in 2012. Human face and hand detection, reputation and tracking are critical research areas for lots laptop interaction packages. A Hybrid Method Using Haar-like and Skin-Color Algorithm for Hand Posture Detection, Recognition and Tracking by S. Bilal, Rina Akmeliawati, Memo Jimi El Salami, Amir A. Shafie, El Mehdi Bouhabba [12] at 2010. The human hand is a complicated articulated item together with many linked parts and joints.

\section{COLOR RECOGNITION SYSTEM}

Color detection is an essential stage in the greater part of the picture handling application, if the application depends on the shading data, for example, street sign identification, and color recognition, skin shading location, object location and article following and so on. The execution of consequent modules in a picture preparing application is unfavourably influenced by the past modules, the precision of shading location with a superior wind up critical in a few applications. This technique used for a productive shading recognition in RGB space utilizing a gathering of specialists in various levelled structure. In this structure, a lot of specialists is allotted to assess R, G, and B segments of a pixel and after that builds a level of enrolment to the arrangement of predefined class of hues for the given pixel. At that point, an ace neural system builds its conclusion dependent on the enrolment probabilities given by the arrangement of specialists. Subjective and quantitative assessments of the outcomes demonstrate that the proposed progressive structure of neural systems is better over the traditional neural system classifier in shading identification.

\subsection{CTS 3200 Color Sensor}

The sensor has four unique sorts of channel secured diodes. In the $8 \times 8$ cluster of photodiodes, 16 photodiodes have Red channels, 16 have Blue channels, 16 have Green channels and the rest 16 photodiodes are clear without any channels. Each sort can be enacted utilizing the S2, S3 determination inputs as shown in Table 1.

Table 1. Pin Configuration of CTS 3200 Color Sensor

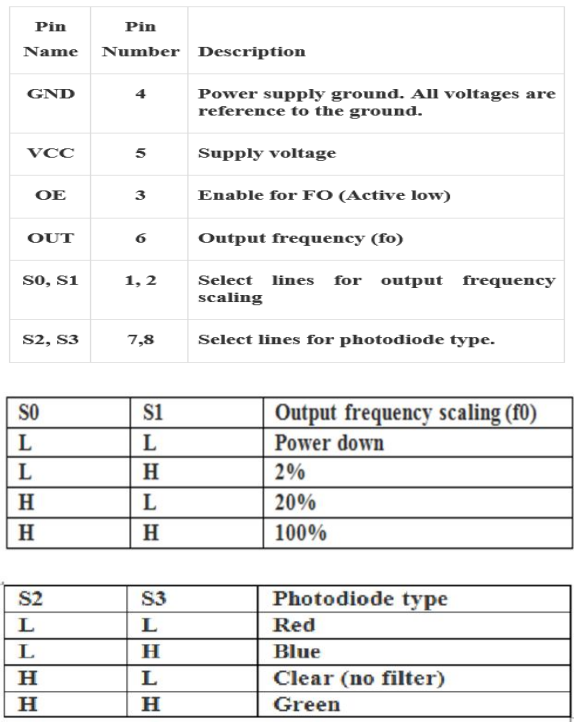

Since each photo diodes are covered with various channels every one of them can distinguish the comparing hues. For instance, while picking the red channel, just red episode light can transverse, blue and green will be forestalled. Estimating the recurrence, we get the red-light force. Correspondingly, when pick different channels blue or green light may appear. Recurrence scaling choice by utilizing the S0, S1 select lines. TCS3200 shading sensor module shown in figure 1 which can be utilized to recognize the colors with the assistance of an any FPGA / Embedded System. As a matter of fact, the microcontroller is estimating the yield recurrence from the sixth stick. To decide the shade of an item, we've to quantify the recurrence from sixth stick when each channel is actuated. Set both S2 and S3 to LOW, measure the recurrence. Presently we get the power of RED segment in the article. Set $\mathrm{S} 2$ to LOW and $\mathrm{S} 3$ as HIGH to get the power of BLUE part in the item. Set both S2 and S3 to HIGH and get the force of GREEN segment in the article. Look at the frequencies of the three parts to get the real shade. Figure 2 shows the Basys 3 board, VGA color test is generated on Basys 3 shown in figure 3. Figure $4 \& 5$ describes sensor interface to FPGA where it 
detects the color and outputs led as indication. Figure 6, 7, 8 shows the simulation results of $\mathrm{R}, \mathrm{G}, \mathrm{B}$ colors as per pin configuration of color sensor shown table 1 .

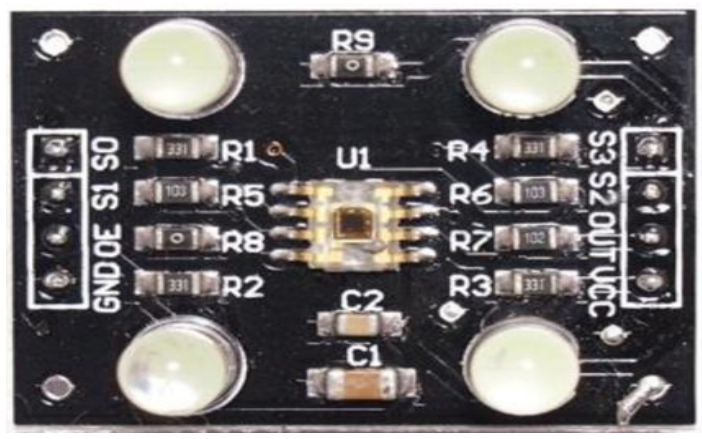

Figure 1: TCS3200 Color Sensor Module

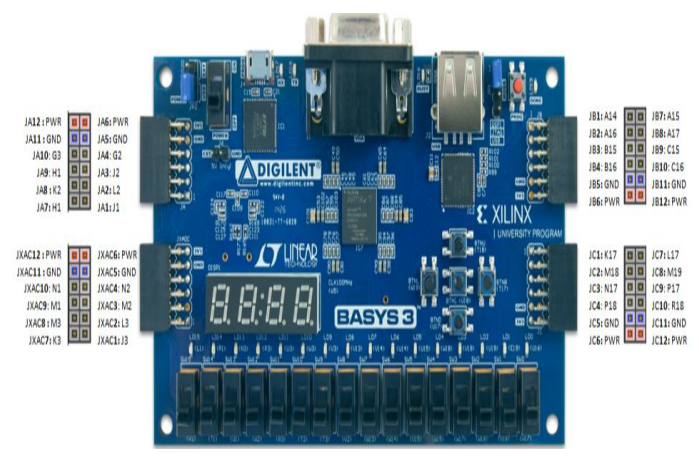

Figure 2: Basys3 FPGA Board

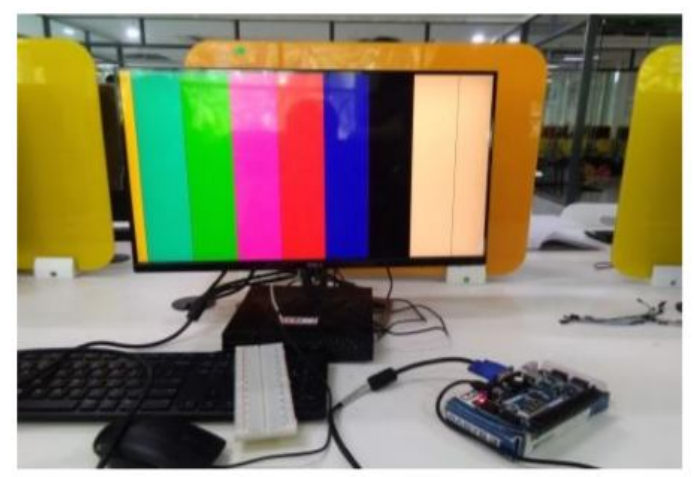

Figure 3: Basys3 FPGA Board VGA test pattern

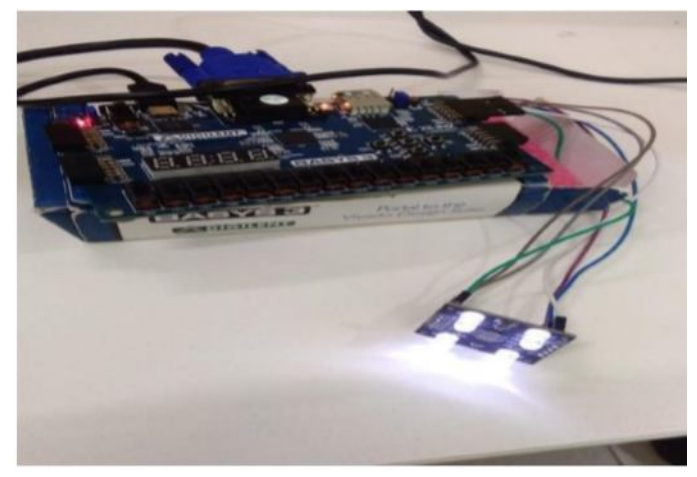

Figure 4: TCS3200 Color Sensor interfaced to Basys3 FPGA

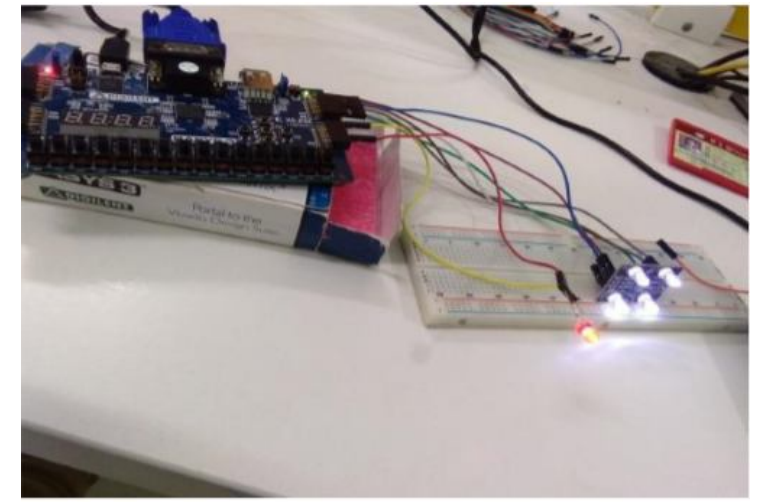

Figure 5: TCS3200 Color Sensor interfaced to Basys3 FPGA with detected output

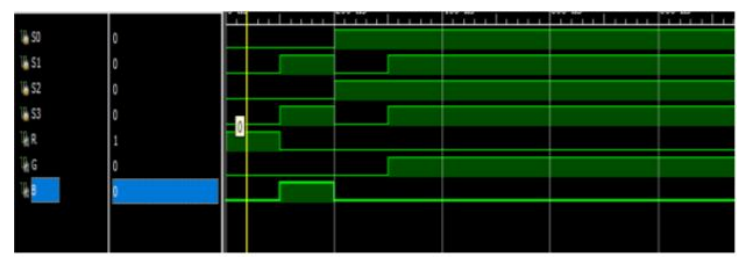

Figure 6: Simulation of output of S2, S3 combination of Red

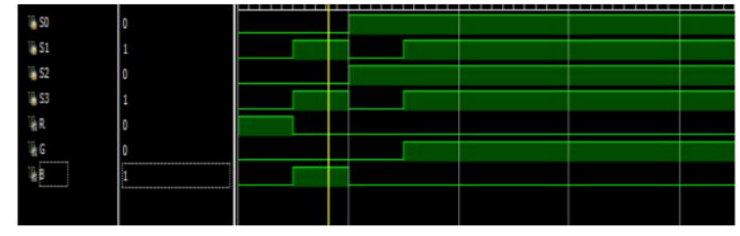

Figure 7: Simulation of output of S2, S3 combination of Blue

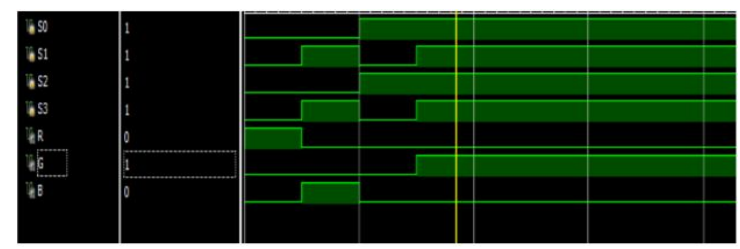

Figure 8: Simulation of output of S2, S3 combination of Green

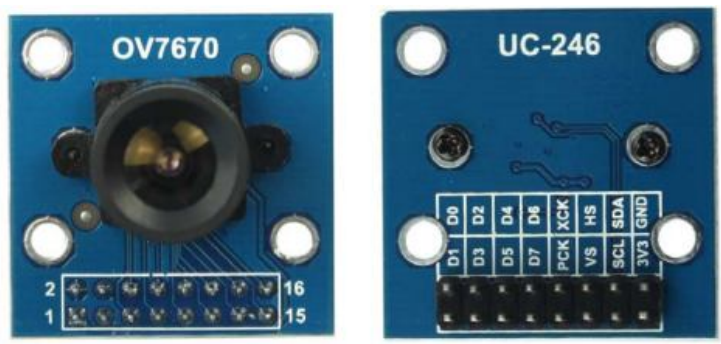

Figure 9: OV7670 Camera Module

Figure 9 shows the Hardware PMOD OV7670 Camera interface Module. Which consists of 16 pins each pins description is shown in Table 2. Block diagram of Color Recognition System is shown in figure 10 consists of camera module Basys3 FPGA Board, VGA cable and required color items for input to camera. 
G.Rakesh Chowdary et al., International Journal of Emerging Trends in Engineering Research, 8(7), July 2020, 3909 - 3914

Table 2: OV7670 Camera Module Pin Configuration

\begin{tabular}{|c|c|c|l|}
\hline Pin No. & PIN NAME & TYPE & DESCRIPTION \\
\hline 1 & VCC & POWER & 3.3v Power supply \\
\hline 2 & GND & Ground & Power ground \\
\hline 3 & SCL & Input & Two-Wire Serial Interface Clock \\
\hline 4 & SDATA & Bi-directional & Two-Wire Serial Interface Data I/O \\
\hline 5 & VSYNC & Output & Active High: Frame Valid; indicates active frame \\
\hline 6 & HREF & Output & Active High: Line/Data Valid; indicates active pixels \\
\hline 7 & PCLK & Output & Pixel Clock output from sensor \\
\hline 8 & XCLK & Input & Master Clock into Sensor \\
\hline 9 & DOUT9 & Output & Pixel Data Output 9 (MSB) \\
\hline 10 & DOUT8 & Output & Pixel Data Output 8 \\
\hline 11 & DOUT7 & Output & Pixel Data Output 7 \\
\hline 12 & DoUT6 & Output & Pixel Data Output 6 \\
\hline 13 & DOUT5 & Output & Pixel Data Output 5 \\
\hline 14 & DOUT4 & Output & Pixel Data Output 4 \\
\hline 15 & DOUT3 & Output & Pixel Data Output 3 \\
\hline 16 & DouT2 & Output & Pixel Data Output 2 (LSB) \\
\hline
\end{tabular}

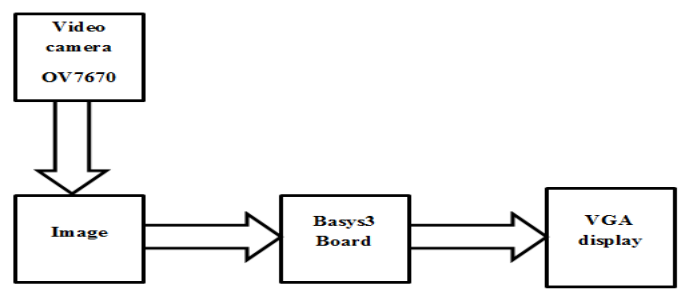

Figure 10: Block diagram of FPGA based Color Recognition System

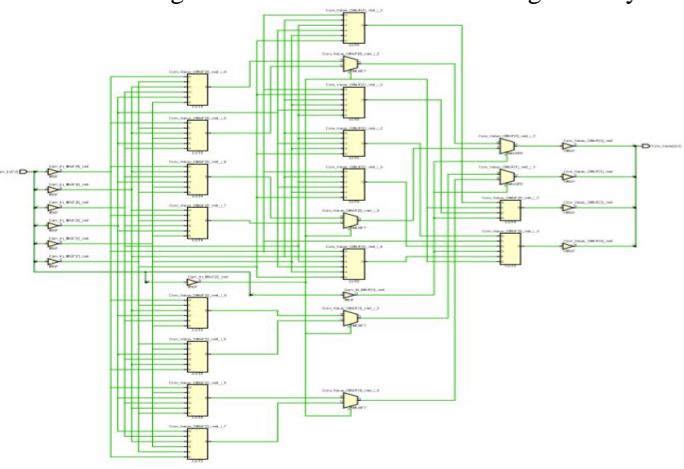

Figure 11: RTL Schematic of OV7670 Interface

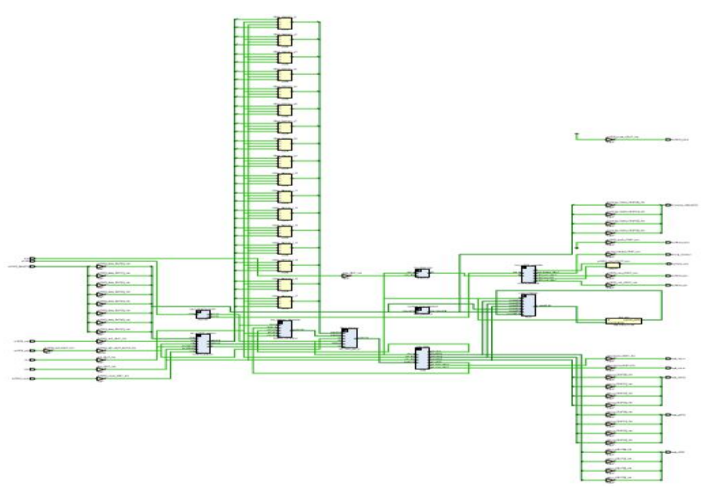

Figure 12: RTL Schematic of OV7670 with VGA Interface

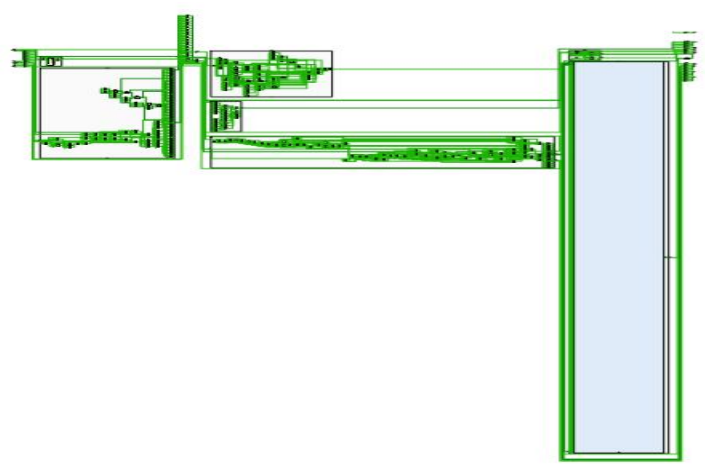

Figure 13: Technology Schematic of FPGA based Color Recognition System with Logic Analyzer

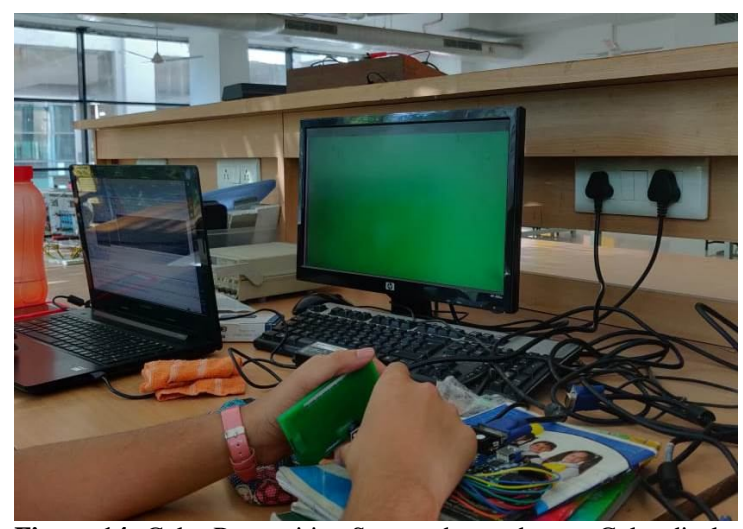

Figure 14: Color Recognition System detected green Color displaying in VGA Monitor

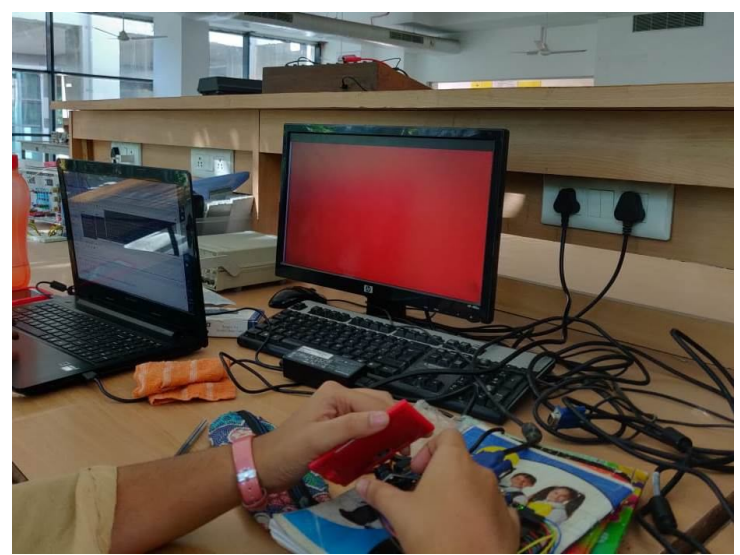

Figure 15: Color Recognition System detected Red Color displaying in VGA Monitor

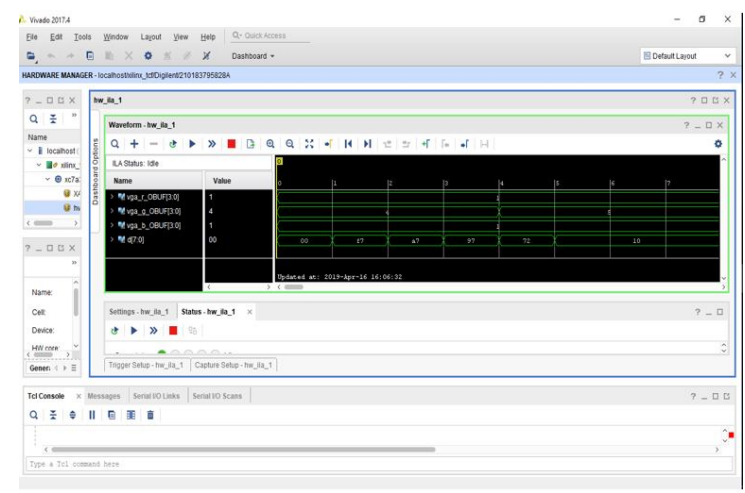

Figure 16: Color Recognition System detected Green Color displaying pixel values in Logic Analyzer 


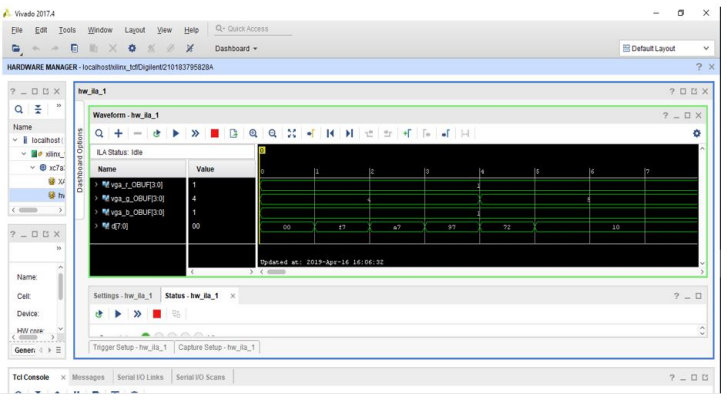

Figure 17: Color Recognition System detected Red Color displaying pixel values in Logic Analyzer

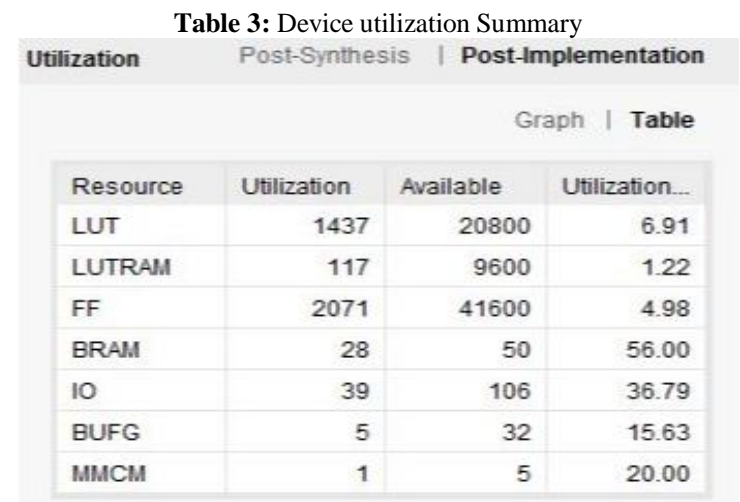

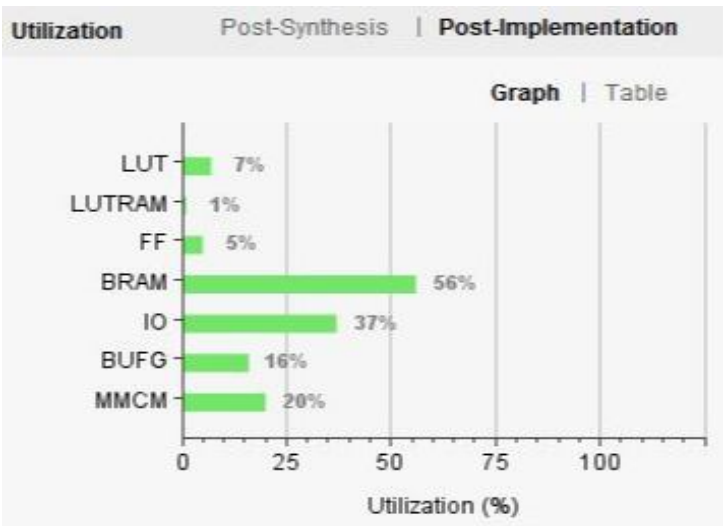

Figure 18: Graphical View of Percentage of Utilized Hardware Resources for Color Recognition System

Figure 11 and 12 shows the RTL Schematic of OV7670 Interface with and without VGA Interface after describing HDL Logic. Technology Schematic of FPGA based Color Recognition System with binded chipscope pro logic analyzer is shown in figure 13 where more number of real input color pixels values are captured whose values are shown in figure 16 green color and red color in figure 17. Figure 14 and 15 shows interfacing basys 3 board with OV7670 camera module. In this system, the camera detects the outputs the hex values of the acquired color and thereby it processes the hex values to FPGA. Then the processes values are displayed in the VGA monitor. Device utilization summary of CRS is shown in Table 3. Post Implementation of CRS graphical view is shown in figure 18 . Colors are most widely used in regular day to day existence at different places like in markets, general stores, college ID cards and groceries. It takes a lot of time and may lead to errors. It is necessary to develop automatic color recognition systems.

\section{CONCLUSION}

Color recognition system is proposed and implemented on FPGA using Verilog HDL. Software simulations are done in Xilinx VIVADO. In real time, color is recognized, hex values of the color are captured using Chip Scope Pro logic analyser and the unique values for each color are sampled at different instant of time. When the color is placed in front of camera and the resulted hex values are matched with the prestored data in FPGA block memory. Then CRS outputs are displayed color on monitor. Depends on the application, usage of block memory may vary for targeted architecture. Due to limited BRAM on Basys3 board only few images can be stored and captured. When camera is interfaced, proper lighting must be there with suitable position, distance of the camera from the color must be fixed to obtain approximate pixel values. An FPGA based color processing along with recognition is done. Many advantages are perceived in designing and deploying with reconfigurable architectures where it provides high processing performance, flexibility, and low development cost. Further research can be extended by improving the speed and accuracy of the system using high resolution cameras to detect the wide range of color shades for huge applications like machine learning and artificial intelligence.

\section{REFERENCES}

1. Kawulok M., Kawulok J., Nalepa J., Smolka B. (2014) Self-Adaptive Skin Segmentation in Color Images. In: Bayro-Corrochano E., Hancock E. (eds) Progress in Pattern Recognition, Image Analysis, Computer Vision, and Applications. CIARP 2014. Lecture Notes in Computer Science, vol 8827. Springer, Cham.

2. M. Kawulok, "Fast propagation-based skin regions segmentation in color images," 2013 10th IEEE International Conference and Workshops on Automatic Face and Gesture Recognition (FG), Shanghai, 2013, pp. 1-7, doi: 10.1109/FG.2013.6553733.

3. Abin, A.A., Fotouhi, M. \& Kasaei, S. A new dynamic cellular learning automata-based skin detector. Multimedia Systems 15, 309-323 (2009).

4. Kawulok M. (2008) Dynamic Skin Detection in Color Images for Sign Language Recognition. In: Elmoataz A., Lezoray O., Nouboud F., Mammass D. (eds) Image and Signal Processing. ICISP 2008. Lecture Notes in Computer Science, vol 5099. Springer, Berlin, Heidelberg.

5. Abbas Cheddad, Joan Condell, Kevin Curran, PaulMc Kevitt, A skin tone detection algorithm for an adaptive approach to steganography Signal Processing, 2009, ISSN: 0165-1684, Vol: 89, Issue: 12, Page: 2465-2478.

6. M. Abdullah-Al-Wadud and O. Chae, "Skin Segmentation Using Color Distance Map and Water-Flow Property," in Information Assurance and 
Security, International Symposium on, null, 2008 pp. 83-88. doi: 10.1109/IAS.2008.65.

7. Beniak, Marzena et al. "Automatic face detection based on chrominance components analysis." 2008 15th International Conference on Systems, Signals and Image Processing (2008): 475-478. https://doi.org/10.1109/IWSSIP.2008.4604469

8. Choudhury, Abhishek et al. "A Novel Skin Tone Detection Algorithm for Contraband Image Analysis." 2008 Third International Workshop on Systematic Approaches to Digital Forensic Engineering (2008): 3-9.

9. W. Zhao, W. and R. Chellapa. "Image-based face recognition: Issues and methods". Retrieved September $1,2008$.

10. Michal Kawulok and Jolanta Kawulok and Jakub Nalepa" Spatial-based skin detection using discriminative skin-presence features", Journal of Pattern Recognition Letters", 2014, volume 41, Page: 3-13. https://doi.org/10.1016/j.patrec.2013.08.028

11. Sara Bilal, Rini Akmeliawati, Momoh Jimoh Salami, and Amir A. Shafie. 2015. Dynamic approach for real-time skin detection. J. Real-Time Image Process. 10, 2 (June 2015), 371-385. DOI: https://doi.org/10.1007/s11554-012-0305-2.

12. Bilal, Sara et al. "A hybrid method using haar-like and skin-color algorithm for hand posture detection, recognition and tracking." 2010 IEEE International Conference on Mechatronics and Automation (2010): 934-939.

https://doi.org/10.1109/ICMA.2010.5588576 